\title{
El papel de las ciudades en el desarrollo sostenible: el caso del programa ciudad 21 en Andalucía (España)
}

\author{
Rocío Llamas-Sánchez \\ Ángeles Muñoz-Fernández \\ Guillermo Maraver-Tarifa \\ Belén Senés-García \\ Facultad de Ciencias Económicas y Empresariales, Universidad de Granada.
}

RESUmen | El Programa Ciudad 21 en Andalucía (España) es un programa que brinda apoyo y asesoramiento a las ciudades y pueblos andaluces que manifiesten su compromiso para crear un plan local hacia la sostenibilidad adoptando una Agenda 21 Local (A21L). La A21L es un programa de actuación a través del cual los ayuntamientos realizan un diagnóstico de sus municipios y tras la realización de un plan de participación ciudadana, definen e implantan una serie de actuaciones que consigan la mejora económica, social y medioambiental de sus municipios. En este trabajo se analizan los factores impulsores y obstaculizadores de la implementación de este programa en los ayuntamientos andaluces. Las conclusiones obtenidas muestran recomendaciones para mejorar estos procesos extensibles a otras regiones que se enfrentan a retos similares para conseguir el desarrollo sostenible de los pueblos y ciudades del mundo.

PALABRAS CLAVE | Desarrollo regional y local, desarrollo sustentable, gestión ambiental, gobierno local.

ABSTRACT | The City 21 Program in Andalusia (Spain) is a program that provides support and advice to cities and towns in Andalusia which demonstrate their commitment to create a local plan for sustainability by adopting Local Agenda 21 (LA21). LA21 is a program of action through which town councils make a diagnosis of their municipalities and, following a citizen participation plan, define and implement a series of interventions to achieve improved economic, social and environmental performance in their municipalities. In this paper, we analyze the drivers and hindering factors in the implementation of this program in the Andalusian municipalities. The conclusions offer recommendations to improve these processes that can be generalized to other regions facing similar challenges in the sustainable development of villages and towns around the world.

KEY WORDS | Regional and local development, sustainable development, environmental management, local government.

Recibido el 11 de junio de 2009, aprobado el 12 de julio de 2010.

Agradecimientos: Los autores agradecen a la Empresa de Gestión Medioambiental, S.A. (EGMASA), empresa pública de la Junta de Andalucía, el apoyo, colaboración y financiación del presente trabajo de investigación. Correspondencia: Universidad de Granada, Facultad de Ciencias Económicas y Empresariales. Campus Universitario de Cartuja, s/n. 18011 Granada, España. Fono: +34952698756, E-mail: rllamas@ugr.es 


\section{Introducción}

En las últimas décadas los temas relacionados con el medio ambiente han suscitado gran interés y preocupación. Tras una época en la que el crecimiento económico olvidaba los límites ambientales, la sociedad empezó a darse cuenta de que esta forma de actuar deterioraba el medio ambiente. En la década de los noventa aumenta la concienciación en organismos, iniciativas gubernamentales y opinión pública en beneficio del medio ambiente. Con ello va naciendo el concepto de desarrollo sostenible definido como "aquel que satisface las necesidades de la generación presente sin comprometer la capacidad de las generaciones futuras para satisfacer sus propias necesidades" (Brundlant, 1987). La búsqueda de un desarrollo territorial integrado debe ser capaz de hacer compatible la competitividad, el bienestar social, la sostenibilidad ambiental y la reducción de los desequilibrios territoriales (Caravaca, González y Silva, 2005) añadiendo, al igual que Winchester (2006), la dimensión espacial (desde la escala local a la global) a las dimensiones económica, social y ambiental.

En la Conferencia de las Naciones Unidas sobre el Medio Ambiente y el Desarrollo, conocida como Cumbre para la Tierra y celebrada en Río de Janeiro en 1992, uno de los acuerdos aprobados por los gobiernos participantes fue el Programa 21, un plan de acción mundial para promover el desarrollo sostenible. En su capítulo 28 y de acuerdo con el principio de subsidiariedad (por el que las autoridades que deben tomar las decisiones referentes a algo en concreto deben ser aquellas que estén en mayor contacto con las personas implicadas), hace un llamamiento a las autoridades locales para que establezcan estrategias de desarrollo sostenible traducidas a planes concretos para una ciudad o pueblo específicos.

La plasmación en Europa de la Cumbre de Río en cuanto a iniciativas locales se reflejó en 1994 en la Primera Conferencia Europea de Ciudades y Poblaciones Sostenibles celebrada en Aalborg (Dinamarca), donde se elaboró una Carta -la Carta de Aalborg- que deben firmar las autoridades locales europeas que decidan comprometerse a participar en el proceso de A21L. En Europa son más de dos mil los ayuntamientos adheridos, comprometidos a implantar una A21L.

En España el mayor impulso proviene de las comunidades autónomas. La comunidad autónoma andaluza, a través del Programa de Sostenibilidad Ambiental Urbana "Ciudad 21", apoya a los ayuntamientos andaluces a adoptar comportamientos que persiguen hacer más sostenibles las ciudades y pueblos de Andalucía.

El Programa Ciudad 21, impulsado por la Consejería de Medio Ambiente de la Junta de Andalucía, y en colaboración con la FAMP (Federación Andaluza de Municipios y Provincias) tiene como objetivo mejorar sustancialmente la calidad del medio ambiente urbano, en el marco de las iniciativas que fomenten un desarrollo sostenible, para lo cual brinda apoyo y asesoramiento técnico a las ciudades andaluzas que manifiesten su compromiso para crear un plan de acción local hacia la sostenibilidad fomentando la participación ciudadana. 
El Programa Ciudad 21 está destinado a todos los municipios de Andalucía de la Red de Ciudades Sostenibles de Andalucía (RECSA) ${ }^{1}$ y es de adhesión voluntaria. El ayuntamiento se compromete a analizar y cofinanciar el diagnóstico, a mejorar la calidad ambiental de su municipio, a establecer herramientas que desarrollen la participación ciudadana y a definir y acometer un plan de acción local. La secuencia del programa sería la siguiente: Diagnóstico Ambiental, Plan de Participación Ciudadana, Plan de Acción Ambiental, Plan de Difusión y Plan de Seguimiento.

En la primera convocatoria se adhirieron 111 municipios de los 770 municipios andaluces, que representaban a casi 5.000.000 de habitantes de los algo más de 7.400.000 habitantes de Andalucía. Posteriormente ha habido una segunda convocatoria donde el número de municipios participantes ha llegado a los 231 .

Interesados por este programa se elaboró una propuesta de investigación con el título "Los municipios andaluces y el desarrollo sostenible: factores condicionantes", que fue acogida favorablemente por la Consejería de Medio Ambiente de la Junta de Andalucía, formalizándose con la firma de un contrato de investigación. El objetivo de esta investigación era hacer un análisis de la situación particular de Andalucía en cuanto al desarrollo sostenible y a la implementación de las A21L.

Fruto de este trabajo, hemos elaborado el presente artículo donde mostramos los principales resultados obtenidos. Así, se analizan los factores impulsores y obstaculizadores de la implementación de este programa con el objetivo de conocer sus repercusiones en los ayuntamientos y municipios andaluces. Para ello, hemos llevado a cabo una investigación cualitativa realizando 13 estudios de caso en 13 de estos ayuntamientos pertenecientes al Programa Ciudad 21. Esta primera parte de la investigación la hemos complementado con un estudio cuantitativo en los 111 ayuntamientos participantes, a través de un cuestionario como instrumento de recogida de información.

La investigación realizada nos ha permitido identificar 11 proposiciones capaces de definir los factores organizacionales y contextuales que influyen en el proceso de adopción de la $\mathrm{A} 21 \mathrm{~L}$, así como las variables que afectan al proceso de innovación de los ayuntamientos.

La cuestión de investigación principal que ha guiado este trabajo es ¿cómo y por qué la innovación se origina, desarrolla, implanta y (quizá) termina en un municipio y en el ámbito del desarrollo sostenible? Esta cuestión supone estudiar la secuencia de decisiones y actuaciones relacionadas con la adopción e implementación de esta innovación. Además, requiere identificar los procesos y estructuras creados que favorecen u obstaculizan los programas de A21L. En definitiva, se pretende conocer por qué fueron tomadas determinadas decisiones en relación con la adopción de la A21L, dónde y por quién fueron tomadas, cómo han sido y están siendo implementadas, qué cambios están produciendo, y con qué resultados, es decir, estudiar un proceso de innovación municipal durante el conjunto de su ciclo de vida.

1 En una primera fase se pensó en municipios de más de 10.000 habitantes y algunos de la RENPA (Red de Espacios Naturales Protegidos de Andalucía). 


\subsection{El desarrollo sostenible como proceso de innovación en los ayuntamientos}

La innovación se ha convertido en un concepto de uso cada vez más frecuente en todo tipo de ámbitos, consolidándose la idea de que un esfuerzo de innovación sostenido resulta ser hoy un factor clave para mejorar la competitividad de las organizaciones y favorecer el desarrollo en los territorios (Méndez, 2002). Los ayuntamientos no están siendo ajenos a esta necesidad de innovación y desarrollo, pues operan en un contexto institucional con presiones hacia el cambio en general y, en estos momentos en particular, hacia actividades encaminadas a la protección del medio ambiente y a la búsqueda de la sostenibilidad.

Nuestra investigación partía asumiendo que la adopción de la A21L supone incorporar una práctica nueva para el contexto específico de cada ayuntamiento $y$, por tanto, innovadora. Una premisa congruente con la siguiente definición de innovación: "La innovación es la secuencia de actividades a través de las cuales un elemento nuevo es introducido dentro de una unidad social, con intención de beneficiar a la unidad, a alguna parte de ella o a la sociedad. El elemento no necesita ser completamente nuevo o desconocido para los miembros de la unidad, pero debe implicar algún cambio perceptible o reto al statu quo" (King, 1992, p. 91). A los efectos de la presente investigación, se puede definir a la A21L como una innovación introducida dentro de una realidad social particular, el ayuntamiento, y que requiere para su implementación una serie de actuaciones que tendrán como finalidad beneficiar al ayuntamiento, a los ciudadanos y al municipio en general.

No sólo nos referimos, por tanto, a la vertiente empresarial de la innovación, sino a la innovación socioinstitucional que "posibilita elevar la capacidad competitiva de empresas y territorios, mejorar la calidad del empleo, maximizar las potencialidades de desarrollo, reducir los riesgos e impactos negativos sobre el medio ambiente, etc." (Caravaca et al., 2005, p. 7).

De acuerdo con Taylor y McAdam (2004), la investigación sobre la adopción e implementación con éxito de una innovación requiere de investigación adicional que permita identificar los procesos y estructuras subyacentes a través de los cuales las organizaciones logran asimilar con éxito una innovación y, finalmente, cambiar. Aunque se han dado importantes pasos en la investigación sobre los procesos de implementación de la innovación, es necesaria más investigación, dado que todavía permanecen abiertas importantes cuestiones (Klein y Knight, 2005).

La implantación de una nueva idea, práctica, programa, producto o servicio en toda organización requiere coherencia en la gestión de multitud de factores que afectan al desarrollo con éxito de la nueva idea y logro de los resultados esperados con el cambio (Muñoz, 2001). En el caso concreto de la A21L como innovación para los ayuntamientos, la identificación y gestión de estos factores hará que se logren resultados sostenibles, pues aunque la idea de lograrlos nazca con fuerza, esa fuerza e impulso inicial de gobiernos, administraciones, autoridades y ciudadanos no está libre de los obstáculos propios que toda idea de cambio genera.

Por otro lado, el contexto también afecta a la implementación exitosa de una innovación. No es posible separar una realidad del contexto donde se genera y realiza. 
"Se debe ver el proceso de cambio organizacional dentro del amplio contexto que incluye el ambiente, que en sí consta de otras organizaciones al igual que patrones y cambios sociales, políticos y económicos, y los mismos esfuerzos de cambio en las organizaciones" (Hall, 1996, p. 212).

\section{Metodología}

Para dar respuesta a nuestra cuestión de investigación optamos por la estrategia de investigación de los estudios de caso. De acuerdo con Yin (1989, p. 13), "los estudios de caso son la estrategia de investigación preferida cuando "cómo" y "por qué" son las cuestiones de investigación formuladas, cuando el investigador tiene poco o ningún control sobre los acontecimientos y cuando el interés recae sobre algún fenómeno coetáneo, dentro de algún contexto de la vida real". Una metodología que permite al investigador estudiar un tópico como un proceso dinámico, no como algo estático (Chetty, 1996). Como señala Gummesson (1988, p. 78), "los métodos de investigación convencional son escasamente aplicables a los procesos de cambio en las organizaciones". El diseño de la investigación ha tenido en cuenta estas dos preocupaciones inherentes en los párrafos anteriores: globalidad y tiempo, y utiliza lo que Yin (1984, p. 41) describe como un diseño de "caso múltiple". Según Eisenhardt (1991, p. 620) "los casos múltiples son un poderoso medio para crear teoría porque permiten replicación y extensión entre casos individuales”.

El método del estudio de caso se puede desarrollar siguiendo dos diferentes enfoques. Yin (1989) usa los estudios de caso para comprobar teoría, mientras que Eisenhardt (1989) usa los estudios de caso para desarrollar teoría. El segundo enfoque ha sido el utilizado en este estudio sobre la implantación de la A21L como innovación por los ayuntamientos de Andalucía. La presente investigación ha tenido, por tanto, carácter exploratorio, es decir, ha buscado identificar -antes que hacer aceptar- a los principales actores, acontecimientos, actividades e influencias que afectan al progreso de la innovación. Para ello, se ha seguido la estrategia de investigación propuesta por Eisenhardt (1989) y que esta autora denomina "desarrollo de teoría desde la investigación de estudio del caso". Un tipo de investigación que pretende desarrollar teoría utilizando estudios de caso y que requiere que el investigador inicie el estudio "tan cerca como sea posible del ideal de ninguna teoría bajo consideración y ninguna hipótesis para probar” (Eisenhardt, 1989, p. 536). Una estrategia de investigación que, dada la riqueza y profundidad de datos que proporciona, continúa siendo aconsejada en los trabajos más recientes sobre adopción e implementación de innovaciones (Taylor y McAdam, 2004).

Aunque no ha sido muy común en los estudios que pretenden "generar teoría", puede resultar valioso especificar, junto a la cuestión de investigación que dirige el estudio, un grupo central de conceptos o variables potencialmente importantes en el proceso de innovación (Eisenhardt, 1989). Esta especificación a priori de conceptos es igualmente relevante para Van de Ven y Poole (1990, p. 317), pues entienden que "implícita o explícitamente, el estudio de cualquier proceso de cambio o innovación requiere un grupo de categorías o variables para describir 
el desarrollo de la innovación”. Si bien, como señala Eisenhardt (1989, p. 536), “a ninguno se le tiene garantizado un lugar en la teoría resultante, no importa cómo de bien sea medido".

Siguiendo la definición del proceso de innovación dada por los investigadores Schroeder, Van de Ven, Scudder y Polley (1989), se han adoptado los cinco conceptos que guiaron la recogida de datos en su estudio: ideas, personas, transacciones, contexto y resultados. Conceptos que fueron seleccionados porque constituyen los factores centrales de interés para los directores de innovación (Van de Ven, 1986), en tanto que "el proceso de innovación consiste en motivar y coordinar personas para desarrollar e implantar nuevas ideas dedicándose a transacciones (o relaciones) con otros y haciendo las adaptaciones necesarias para lograr los resultados deseados dentro de contextos institucionales y organizacionales cambiantes" (Van de Ven y Poole, 1989, p. 317). Además, y como recoge la anterior definición del proceso de innovación, el contexto donde el desarrollo de la innovación tenga lugar afectará a su implementación exitosa. Es decir, no es posible separar una realidad del contexto donde se genera y realiza. "Se debe ver el proceso de cambio organizacional dentro del amplio contexto que incluya el ambiente, que en sí consta de otras organizaciones al igual que patrones y cambios sociales, políticos y económicos, y los mismos esfuerzos de cambio en las organizaciones" (Hall, 1996, p. 212). Por ello, y con objeto de contribuir a la discusión sobre la influencia del contexto sobre la implantación de la innovación, se adoptaron, además, dos conceptos teóricos generales: "contexto interno" y "contexto externo", los cuales pueden afectar a priori al despliegue con éxito de la innovación. De acuerdo con Tolbert y Zucker (1996), si la innovación tiene éxito entre los primeros ayuntamientos que adoptan el programa, la práctica se difundirá entre el resto de municipios y se producirá la institucionalización de la misma. Esta institucionalización se verá afectada tanto por el contexto institucional externo como por el contexto interno (Kostova y Roth, 2002).

Finalmente, consideramos que el estudio nos permitirá conocer la repercusión de la A21L sobre ayuntamientos y ciudades, y valorar en qué medida se está logrando el cambio en un ayuntamiento determinado y en su entorno. En la literatura sobre cambio e innovación se contemplan dos variables de medida de resultados que nos permitirán conocer las diferencias entre ayuntamientos: la eficacia en la implementación y la eficacia de la innovación o, en otras palabras, los beneficios que una organización recibe como resultado de la implementación de una innovación dada (Klein y Sorra, 1996; Klein et al., 2001). La eficacia en la implementación es una condición necesaria, aunque no suficiente, para la eficacia de la innovación; es decir, la implementación eficaz de una innovación dada no garantiza que la innovación tendrá el efecto pretendido sobre la organización adoptadora (Klein et al., 2001).

El siguiente gráfico recoge el conjunto de variables seleccionadas como potencialmente importantes en el proceso de innovación, dando cierta estructura a este estudio (Figura 1). 
FIGURA 1 | Conjunto de variables que definen y condicionan el proceso de innovación

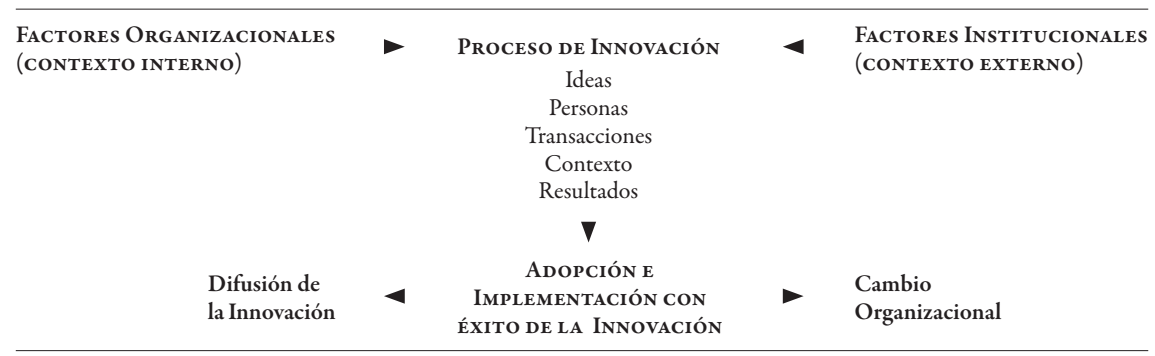

FUENTE ELABORACIÓN PROPIA.

\subsection{Recogida de datos}

El diseño de la recogida de datos estuvo organizado de acuerdo al objetivo de estudiar el proceso de innovación desde su inicio hasta su culminación. Una fortaleza del método de estudio de caso es que los datos se pueden obtener desde una variedad de fuentes, cualitativas y cuantitativas. Para este estudio se utilizaron entrevistas, observación directa, documentación escrita generada por el ayuntamiento durante el proceso, un conjunto de fuentes secundarias y, finalmente, cuestionarios dentro de la lógica de la triangulación en la investigación (Eisenhardt, 1989, p. 538). Destacamos, además, la participación de múltiples investigadores, que aumenta el potencial creativo del estudio y la convergencia de las observaciones por parte de estos investigadores, aumentando la confianza en los resultados (Eisenhardt, 1989).

Describimos a continuación las tres fases en las que se dividió la recogida de datos para este estudio.

La primera fase consistió en recopilar y analizar la información de gran número de fuentes documentales para asegurar que partíamos del estado actual en el que se encontraban el Programa Ciudad 21 y los municipios andaluces. Para ello, también resultaron fundamentales las conversaciones mantenidas con la Secretaría Técnica del Programa Ciudad 21 que facilitó en todo momento documentación y apoyo.

La segunda fase fue la de investigación exploratoria in situ del estado de desarrollo de la A21L en trece ayuntamientos: Vícar, Jerez de la Frontera, San Roque, Montilla, Montoro, Granada, Motril, Cartaya, Punta Umbría, Villacarrillo, Fuente de Piedra, Arahal y El Viso del Alcor. En cada uno de estos ayuntamientos se realizaron entrevistas en profundidad a los responsables de la A21L. En los 13 ayuntamientos visitados han estado representadas las 8 provincias andaluzas. Se trata, además, de municipios de diferente número de habitantes. Uno de los municipios tiene menos de 5.000 habitantes, tres de ellos más de 50.000 habitantes y nueve tienen entre 5.000 y 50.000 habitantes, como muestra la Figura 2. 
FIGURA 2 | Mapa de localización de las entrevistas en profundidad realizadas, ayuntamientos adheridos al Programa Ciudad 21 y cuestionarios recibidos

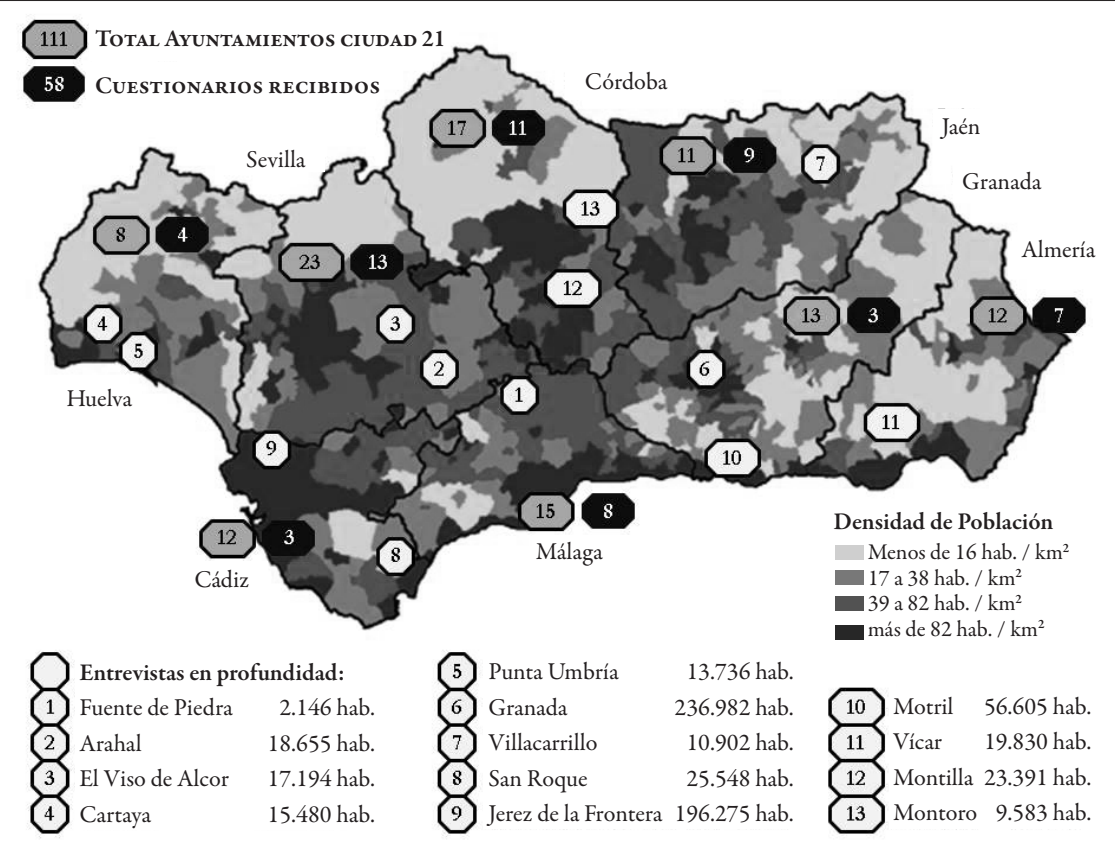

FUENTE ELABORACIÓN PROPIA A PARTIR DE LA INFORMACIÓN APORTADA POR EL INSTITUTO DE ESTADÍSTICA DE ANDALUCÍA MUNICIPIOS ANDALUCES. DATOS BÁSICOS 2007 (WWW.JUNTADEANDALUCIA.ES/IEA/).

Todas las entrevistas fueron realizadas directamente por los responsables del proyecto. El modelo concreto de entrevista quedó configurado bajo el formato de entrevista mixta, con preguntas tanto abiertas (dejando libertad de expresión), como cerradas (con respuestas fijas y detalladas).

En la selección de las preguntas acudimos, en muchos casos, a otros trabajos sobre procesos de sostenibilidad y Agenda 21, por ser investigaciones rigurosas y de gran utilidad, además de facilitar la comparación temporal de determinadas variables. Utilizamos el informe sobre el Estado del Arte de las A21L en España de 2003 realizado por EGMASA y el cuestionario que el ICLEI elaboró y envió en 2002 a ayuntamientos de todo el mundo para medir el progreso de la A21L. También se utilizaron otros estudios de Nuria Font y Joan Subirats de la Universidad Autónoma de Barcelona sobre Agenda 21. No obstante, ninguno de estos trabajos se centraba en Andalucía como población objetivo, ni en el Programa Ciudad 21, por lo que una de las aportaciones básicas de esta investigación es precisamente que aborda la situación andaluza.

En la tercera fase recurrimos a una investigación de mayor alcance y amplitud, usando el cuestionario como instrumento de recogida de información. En este caso el objetivo era acceder a los 111 ayuntamientos adheridos al Programa Ciudad 21. 
Ciertamente la muestra era suficientemente amplia y representativa. El objetivo era conseguir un mayor apoyo y validar con más casos la teoría sobre la implantación de la A21L que el análisis de los trece primeros casos estaba generando. El cuestionario contenía variables relevantes de la primera fase de investigación, a las que añadimos otras nuevas variables obtenidas tras el análisis y valoración de las entrevistas en profundidad realizadas en la segunda fase del estudio.

El cuestionario se dividió en cuatro bloques. El primer bloque incidía en el proceso seguido por el ayuntamiento en la implantación de la $\mathrm{A} 21 \mathrm{~L}$, midiendo aspectos como los objetivos perseguidos con la implementación, la metodología utilizada o la participación ciudadana, entre otros. El segundo bloque profundizaba en los resultados de la A21L, tratando de conocer el grado de familiaridad y experiencia de cada ayuntamiento en relación con las actividades, acontecimientos y dinámica de un proceso como el de la $\mathrm{A} 21 \mathrm{~L}$. El tercer bloque trataba de medir el contexto interno de cada ayuntamiento mediante conceptos como el apoyo a la implementación de los máximos responsables del ayuntamiento, el clima percibido por dichos responsables y la receptividad al cambio. Variables que, como detallaremos más adelante, se fueron definiendo tras los análisis de las entrevistas realizadas en la segunda fase de la investigación. Es decir, este apartado examinaba los posibles antecedentes y consecuencias de la implantación de la A21L en los municipios adheridos al Programa Ciudad 21. Finalmente, se incluía un cuarto bloque con preguntas de carácter general.

Utilizamos para la medición escalas tipo likert de siete puntos, desde el 1 -desacuerdo total- hasta el 7 -acuerdo total-, siendo el 4 la posición de indiferencia. Seguimos las recomendaciones y reglas generales que maximizaran el nivel de respuesta y minimizaran el error (Rodríguez del Bosque, 1999). En concreto, el diseño del cuestionario siguió directrices de textos de investigación de mercados.

Los cuestionarios recibidos, repartidos por provincias, pueden consultarse en la Figura 2.

Se recibieron 58 cuestionarios hasta la fecha límite de recepción, con un 52,25\% de tasa de respuesta. El tamaño de la muestra lo conformaron los 58 cuestionarios recibidos de los 111 municipios adheridos al Programa Ciudad 21 en Andalucía como universo de la población. Para este tamaño muestral de 58, un nivel de confianza del $95 \%$ y $p=q=0,5$, el error muestral que se comete es del $9 \%$. El trabajo de campo se realizó durante 2006, con un primer envío de los cuestionarios en octubre, un reenvío en noviembre y un seguimiento telefónico de la investigación en diciembre de ese mismo año.

\subsection{Análisis de datos}

El análisis de los datos es la parte más difícil y menos codificada para la generación de teoría desde los estudios de caso. A diferencia de la investigación de prueba de hipótesis, la investigación inductiva carece de un modelo generalmente aceptado de análisis, reconociéndole cierta creatividad (Eisenhardt y Bourgeois, 1988, p. 741). Siguiendo una de las tácticas propuestas por Eisenhardt (1989), 
usamos en primer lugar, y para analizar la información obtenida con las entrevistas, el siguiente enfoque: primero, y con objeto de conocer cada municipio individualmente, se redactó una historia de caso sobre el progreso de la A21L en cada uno de los ayuntamientos estudiados. Esto permitió identificar unos patrones de desarrollo específicos de cada ayuntamiento. Segundo, y con objeto de buscar patrones "intercaso", se procedió a organizar la información incluida en las historias de caso de acuerdo con los cinco conceptos que guiaron la recogida de datos en el estudio: ideas, personas, transacciones, contexto y resultados. Después, se realizó un análisis "cross-caso" a fin de buscar similitudes y diferencias entre los casos.

El análisis de estos primeros datos se llevó a cabo a través de grupos de discusión. Primero, los cuatro investigadores intercambiaron opiniones, buscando y definiendo los patrones de desarrollo de la innovación que se desprendían de los datos; segundo, los investigadores mantuvieron reuniones con la Secretaría Técnica del Programa Ciudad 21 con objeto de obtener un mayor consenso respecto a cómo y por qué la innovación se inicia e implementa por parte de los diferentes ayuntamientos.

Después de buscar similitudes y diferencias entre los casos, el proceso que sigue es altamente iterativo, pues consiste en comparar sistemáticamente la estructura que empieza a emerger con la evidencia de cada caso. El objetivo es comparar continuamente teoría y datos con el objeto de ir definiendo el conjunto de proposiciones que dará forma a la teoría que mejor se ajuste a los datos (Eisenhardt, 1989). Después de muchas iteraciones entre datos y teoría, se procedió a la comparación de la teoría resultante con la literatura existente con objeto de dar valor a nuestros resultados. "Vincular la teoría emergente con la literatura ya existente favorece la validez interna, la posibilidad de generalización y el nivel teórico de la teoría generada con el estudio de caso" (Eisenhardt, 1989, p. 545). La tercera fase de la recogida de información nos permitiría reforzar la validez de la nueva teoría generada con los estudios de caso.

Para el tratamiento de la información obtenida con el cuestionario en la tercera fase de la investigación se utilizó el paquete estadístico DYANE versión 2. Para informatizar los datos se diseñó una base al efecto. Para evitar posibles errores que pudieran introducir sesgo en la investigación se realizó un control sobre la mecanización y se revisó un tercio de los cuestionarios. El análisis de los datos se inició con un estudio de la muestra mediante tablas de frecuencias relativas a las cuestiones de carácter general, así como sobre el resto de cuestiones planteadas en el cuestionario. Adicionalmente se realizaron diferentes test F de Snedecor para determinar si los valores medios obtenidos en algunas de las categorías de las variables del cuestionario eran significativamente distintos dependiendo: 1) del nivel de conocimiento sobre sostenibilidad; 2) de si la A21L operaba de forma paralela o integrada a la gestión municipal; 3) de si los entrevistados percibían que las autoridades andaluzas realizaban sus procesos de forma unilateral o coordinada; 4) de si se percibía la A21L como un cambio radical o incremental; 5) de que el nivel de implementación de la A21L estuviera por encima del $70 \%$ o por debajo del 30\% de las actuaciones definidas en el plan de acción, y 6) de que los resultados 
de la implementación conseguidos con la A21L estuvieran por encima del 70\% o por debajo del $30 \%$ de eficacia.

\section{Presentación de resultados: proposiciones}

La investigación realizada nos ha permitido identificar dos grupos de proposiciones en función, por un lado, de los factores contextuales y organizacionales que influyen en el desarrollo del proceso de A21L y, por otro, de las variables que influyen en el proceso de innovación en los ayuntamientos. Iniciamos este apartado recogiendo las proposiciones relacionadas con el contexto externo de los ayuntamientos, a continuación las relativas a los factores organizacionales o internos y, finalmente, recogemos el grupo de proposiciones que recogen las variables que afectan al proceso de la innovación.

\section{Proposición 1: las presiones coercitivas, normativas y miméticas del contexto ins- titucional de los ayuntamientos del Programa Ciudad 21 ejercen influencia sobre los procesos de adopción de esta innovación}

La implantación de la A21L por los ayuntamientos estudiados transciende el interior de estas organizaciones y encuentra las principales motivaciones para su adopción en el contexto institucional de los ayuntamientos. Dentro de la teoría de la organización, la teoría institucional define y analiza las diferentes presiones del contexto institucional que deben afrontar las organizaciones y a las que tienen que dar respuesta. Estas presiones coercitivas, normativas y miméticas (DiMaggio y Powell, 1983) inciden más en las organizaciones públicas, por ser éstas las que se enfrentan a entornos altamente institucionalizados y porque dependen de la opinión pública para obtener legitimidad (Dobbin et al., 1993).

Debido a los procesos coercitivos, normativos y miméticos, las organizaciones acaban adoptando ciertas estructuras, programas, políticas y procedimientos (DiMaggio y Powell, 1983; Greenwood et al., 2002; Meyer y Rowan, 1977; Scott, 1987). En cuanto a la presión coercitiva, podemos destacar el incremento de la legislación en materia medioambiental que, cada vez más y con más exigencia, regula las actividades de las empresas y de las organizaciones públicas para la protección del medio ambiente. Sin embargo, la A21L no es una práctica de obligado cumplimiento, ya que los ayuntamientos deciden adoptarla libremente, lo cual no quiere decir que no haya una presión coercitiva implícita, no tanto por las sanciones de no implantarla (que no las hay), sino por las expectativas de recibir apoyo al hacerlo. Por su parte, la presión normativa incluye valores y normas que se traducen en determinadas prácticas y políticas. En este caso, aun cuando en las últimas décadas los valores de desarrollo sostenible se han difundido en diversas esferas, en muchas ocasiones son los propios responsables o participantes de la A21L los que difunden valores que derivan de su propia formación profesional (ya sea en la universidad o en otros centros, o ya sea de la formación posterior o asistencia a cursos, seminarios, jornadas, etc.), valores considerados legítimos y las prácti- 
cas encaminadas a conseguirlos, las apropiadas. Por último, la presión mimética deriva de establecer como modelos a otras organizaciones que se perciben como exitosas. Así, "algunas ciudades son elegidas como referencias modeladoras, y sus programas y proyectos incorporados en la agenda urbana hegemónica” (Sánchez y Moura, 2005, p. 21). Los ayuntamientos que no habían adoptado la A21L han podido conocerla por otros ayuntamientos que la han adoptado, y éste puede ser un factor impulsor de su adopción.

Estos tres tipos de presiones institucionales cambian el campo organizativo del ayuntamiento, puesto que al ser presiones cambiantes (aparecen nuevas leyes que persiguen una mayor sostenibilidad, valores sociales a favor del desarrollo sostenible, difusión de prácticas exitosas relacionadas con el medio ambiente, etc.), obligan a los ayuntamientos a responder a las mismas y a responder a los requerimientos sociales de ciudadanos y grupos de interés con los que el ayuntamiento crea redes sociales. Estas presiones afectan positivamente al mayor grado de inmersión del ayuntamiento en su contexto institucional (Granovetter, 1985).

Como los investigadores han podido verificar, la motivación más generalizada a la hora de implantar la A21L por parte de los ayuntamientos ha sido el desarrollo de una política ambiental adecuada. La idea de adoptar la A21L y de participar en el Programa Ciudad 21 surge de los técnicos del ayuntamiento, por lo que la profesionalización, característica de la presión normativa (DiMaggio y Powell, 1983), se vislumbra como un vehículo transmisor de los valores de la sostenibilidad. Estos técnicos, así como los cargos políticos, suelen pertenecer a redes de municipios, redes de sostenibilidad ambiental, etc. Los ayuntamientos que forman parte del Programa Ciudad 21 pertenecen a la FAMP y a la RECSA, entre otras. También, la mayoría de ayuntamientos participa en jornadas y seminarios ad hoc, por lo que la presión normativa para implantar estas prácticas sostenibles se siente cada vez más.

Por otro lado, todos los ayuntamientos tenían muy presente el aumento de la legislación actual sobre materia medioambiental, alertados sobre nuevas prácticas y comportamientos sostenibles quizás no sólo por evitar un castigo o sanción, sino por las posibles recompensas. Entre las motivaciones que impulsaban a adoptar la A21L se aludía repetidamente a la subvención que reciben por adherirse al Programa Ciudad 21, demostrando la presión coercitiva.

En cuanto a las presiones miméticas, los ayuntamientos mostraron conocer las experiencias exitosas de otros ayuntamientos. Los medios de comunicación juegan también una labor importante, puesto que el Programa Ciudad 21 goza de gran publicidad. Aun así, pensamos que conforme el número de ayuntamientos que forman parte de este programa vaya elevándose, la presión mimética aumentará hasta que esta práctica se perciba como la forma correcta de actuar, quedando entonces completamente institucionalizada (Tolbert y Zucker, 1996). Se pueden observar estos resultados en la figura siguiente (Figura 3). 
FIGURA 3 | Factores del contexto que influyeron en la adopción de la A21L

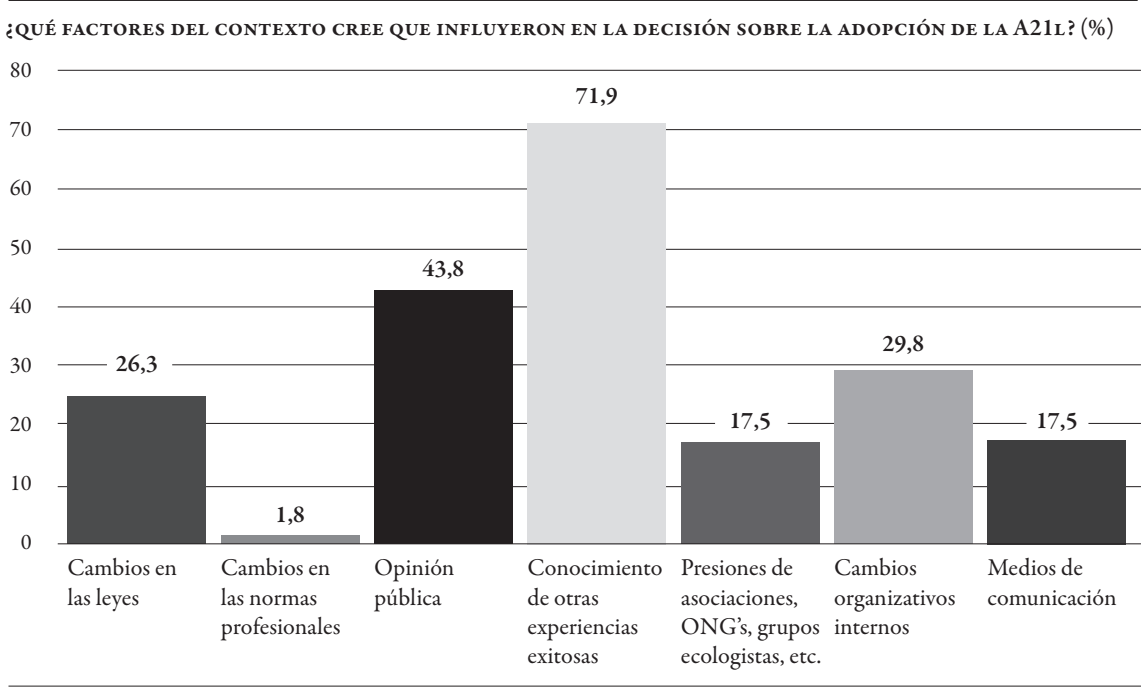

FUENTE ELABORACIÓN PROPIA.

Proposición 2: el tamaño de los ayuntamientos del Programa Ciudad 21 no afecta a la decisión de adoptar esta innovación, sin embargo, según nuestros resultados, los ayuntamientos de menor tamaño son más eficaces que los grandes en la implementación de la A21L

En la investigación sobre innovación no se ha encontrado consistencia al relacionar el tamaño con la innovación (Damanpour, 1992), un debate que continúa abierto. De acuerdo con Rogers (1983), el tamaño es una variable que puede medirse con facilidad y con precisión; además, puede acompañarse de una combinación de factores (recursos, slack o disponibilidad de recursos más allá de las necesidades para el mantenimiento de la organización, estructura organizacional) que podrían promover la innovación (Rogers, 1983). Sin embargo, las organizaciones pequeñas pueden ser más innovadoras por su mayor flexibilidad y capacidad para adaptarse y mejorar (Damanpour, 1992). Asimismo, requieren menos comunicación, menos coordinación y menos influencia para reunir el apoyo necesario, lo que facilita la implementación de innovación (Nord y Tucker, 1987). Otras investigaciones, sin embargo, han enfatizado en el tamaño medio, al encontrar una relación en forma de $U$ invertida entre tamaño de la organización e innovación, es decir, el tamaño estimularía la innovación hasta un punto a partir del cual la organización llegaría a ser tan grande que el crecimiento adicional no estimularía el cambio (Hitt et al., 1990).

De acuerdo con Damanpour (1992), se pueden contemplar dos formas de medir el tamaño del ayuntamiento: como factor organizacional y como factor contextual. En el primer caso el tamaño mide el número de empleados del ayuntamiento y, en el segundo, el número de habitantes de la comunidad o ciudad. En el presente estudio el tamaño se consideró un factor organizacional. 
Según los resultados de la presente investigación, no se han observado diferencias significativas de acuerdo al tamaño de los municipios, sin embargo, se ha constatado que el tamaño menor favorece la implementación de los planes de acción. Según los resultados, los municipios con menos de 15.000 habitantes han implementado significativamente más acciones de las contempladas en el plan de acción que los más grandes (aquéllos con más de 50.000 habitantes). El tamaño del municipio ha demostrado estar asociado también con otras variables estudiadas. Así, los municipios más pequeños ven menos problemas para alcanzar acuerdos con los actores sociales, percibiendo más apoyo del equipo de gobierno que los más grandes. Sin embargo, en los primeros se percibe menos autonomía sobre el trabajo que en los más grandes, para los cuales la autonomía es mayor. La autonomía puede ser una consecuencia directa del tamaño, si bien los resultados no muestran la asociación de mayor tamaño con mayor innovación. Conviene reflexionar sobre si la falta de apoyo del equipo de gobierno en los ayuntamientos más grandes puede estar vetando la autonomía otorgada. El apoyo de los máximos responsables de una organización para la implementación con éxito es un resultado consistente a través de los trabajos de investigación sobre este tópico, presente como se ha comprobado en los ayuntamientos más pequeños y con mayor grado de implementación. Por último, el tamaño parece estar asociado con la culminación de etapas del proceso. Así, los ayuntamientos más grandes inician procesos de evaluación y seguimiento en mayor medida que los pequeños. Esto puede indicar que si bien los ayuntamientos pequeños pueden tener condiciones favorecedoras para la implementación de acciones, flexibilidad, mejor comunicación, apoyo del equipo de gobierno, etc., su menor estructura organizativa les impide o dificulta poner en marcha procesos de evaluación y seguimiento (ver Figura 4).

FIGURA 4 | Evaluación y seguimiento en función del tamaño

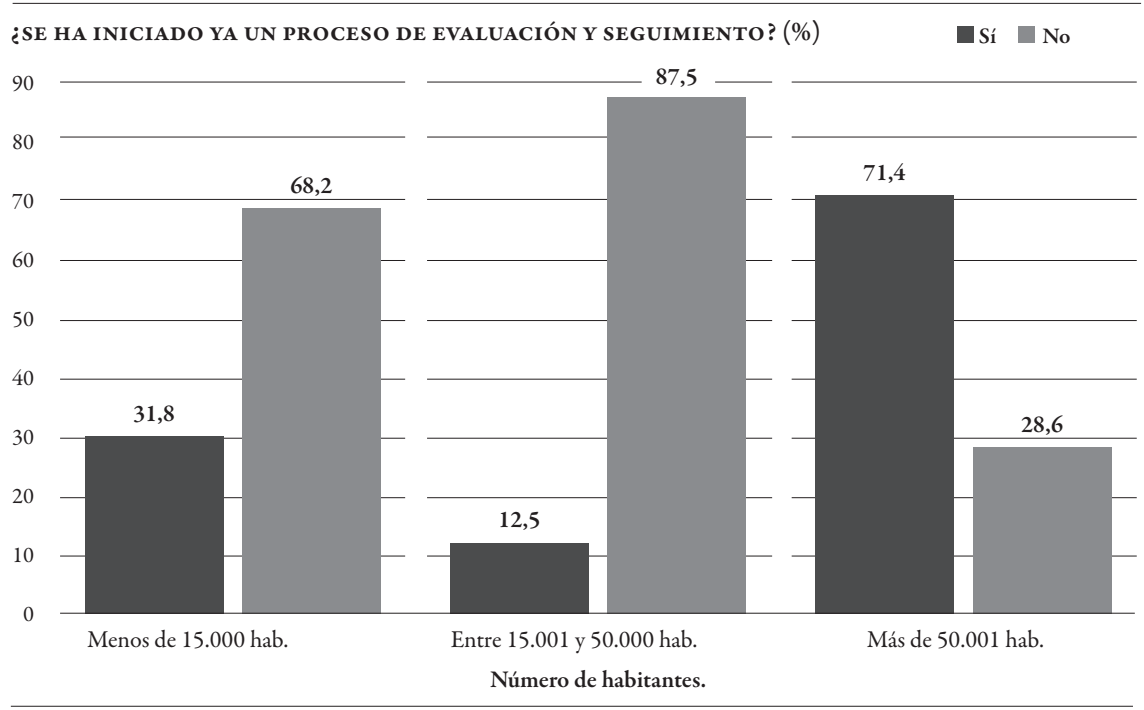

FUENTE ELABORACIÓN PROPIA. 
Proposición 3: la receptividad al cambio en los ayuntamientos del Programa Ciudad 21 favorece la implementación de la innovación

Como señalan Beckhard y Harris (1987), la resistencia al cambio es algo normal en todo proceso de transformación. Las organizaciones son "fuerzas estabilizadoras" (Klein y Knight, 2005) que estarán abiertas al cambio y lo valoran como un objetivo en diferentes grados (Holahan et al., 2004). Siguiendo a estos últimos autores, hemos recogido la variable "receptividad al cambio", en tanto que "las organizaciones con elevada receptividad valoran el cambio, la experimentación y el hacer las cosas de manera diferente. Así, estas organizaciones pueden tener normas o estructuras que les permitan implementar nuevas políticas y prácticas que apoyen la implementación de innovación más fácilmente y ajusten estas políticas y prácticas conforme sea necesario" (Holahan et al., 2004, p. 36). En efecto, la A21L supone que los ayuntamientos y su personal incorporen nuevos criterios a la hora de tomar decisiones, nuevas prácticas y actuaciones en el ayuntamiento, así como que se incorpore nuevo personal con conocimientos especializados en la misma. El éxito en la implementación de la A21L requerirá de entornos receptivos a los cambios, capaces de anticipar y gestionar posibles resistencias, donde se perciban "ganas" y entusiasmo en el nuevo proyecto. Como se señaló anteriormente, los ayuntamientos de esta investigación tienen en común que todos se encuentran en fase de implementación de la $\mathrm{A} 21 \mathrm{~L}$, fase que presenta la mayor resistencia a los cambios (Pardo del Val y Martínez, 2005, p. 47), y donde es más necesario aún que los miembros del ayuntamiento "se crean la agenda", como manifestaba uno de los entrevistados.

En la presente investigación, la receptividad al cambio se ha definido de acuerdo a las percepciones del entrevistado sobre aspectos relacionados con la A21L que se han adoptado sin resistencia, y sobre si se percibe o no al ayuntamiento como flexible y abierto al cambio. La mayoría de entrevistados manifiesta que la adopción de la A21L no se realizó con una considerable resistencia sino con entusiasmo, describiéndose al ayuntamiento como flexible y adaptándose al cambio continuamente. Un 70\% de los ayuntamientos estuvo en desacuerdo con la afirmación de que la adopción de la A21L se había realizado con una gran resistencia al cambio. Al contrario, un 60\% reconoció que se había hecho con gran entusiasmo. No ven que el ayuntamiento tenga más interés por mantener las cosas tal y como están, que por el cambio. En definitiva, los ayuntamientos que han adoptado la A21L muestran alta receptividad al cambio.

\section{Proposición 4: el clima de la implementación de la innovación en los ayuntamien-} tos del Programa Ciudad 21 influirá positivamente en el éxito de la implementación de la A21L

"El clima es la atmósfera creada por determinadas prácticas, procedimientos o recompensas que los empleados perciben en sus organizaciones. Estas percepciones se van desarrollando día a día. No se basan en lo que la dirección, la hoja informativa de la organización o el informe anual publicitan, sino en el comportamiento de los directivos y en las acciones que recompensen" (Schneider et al., 1994, p. 18). Luego, los responsables de una organización crean un clima específico por lo que hacen, no por lo que dicen. 
En efecto, los empleados observan lo que les ocurre a ellos y en su entorno, sacan conclusiones acerca de las prioridades de su organización y configuran sus propias prioridades de acuerdo con ello. Así, dirigen y orientan sus energías y sus competencias en un proceso en el que se está creando el clima, de acuerdo con el concepto aportado por Klein y Sorra (1996, p. 1060), quienes afirman que el clima es el "resultado de las experiencias y observaciones compartidas por los empleados y la información y discusiones que desarrollan acerca de las políticas y prácticas de implementación en la organización". De acuerdo con estos autores, "cuanto más comprehensiva y consistentemente sean percibidas las políticas y prácticas por los empleados, fomenten, nutran y recompensen el uso de una innovación dada, más fuerte será el clima para la implementación de la innovación" (Klein y Sorra, 1996, p. 1060). Si los trabajadores responsables de la implementación de la A21L y aquellos afectados por ella perciben que ésta es una prioridad en el municipio, si perciben los esfuerzos realizados por éste para alcanzar el éxito, si consideran que la implementación de la A21L se ha convertido en un objetivo importante y si, además, perciben preocupación y empuje por aprovechar al máximo la $\mathrm{A} 21 \mathrm{~L}$, todo ello irá creando el clima adecuado para impulsar el proceso de implementación de la A21L, tanto mejor cuanto más se hayan discutido y compartido dichas percepciones.

En esta investigación el clima de implementación ha quedado relacionado con aspectos como la prioridad, el esfuerzo, el compromiso y el empuje del ayuntamiento. Se considera que la implementación de la A21L tiene alta prioridad para el ayuntamiento y que uno de los objetivos del mismo es utilizar la A21L de una manera eficaz, preocupándose por el éxito de esta práctica.

\section{Proposición 5: en los ayuntamientos del Programa Ciudad 21, el apoyo del equipo de gobierno mejora el clima de implementación de la A21L}

El apoyo a la implementación es una variable relacionada con el clima. La literatura sobre cambio organizativo e innovación (Angle y Van de Ven, 1989; Beer, 1988; Klein y Sorra, 1996; Nadler y Tushman, 1989) revela que el apoyo del equipo de gobierno a la innovación es el principal antecedente del clima favorecedor de la implementación. Sin embargo, en muchas ocasiones se fracasa al dar este apoyo. Klein y Sorra (1996) proponen dos motivos de fallo: en primer lugar, no dejar participar a personas de más bajo nivel jerárquico dentro del ayuntamiento; en segundo lugar, carecer de un conocimiento profundo sobre la innovación. Centrándonos en este segundo motivo (sobre el primero haremos referencia cuando hablemos de la participación), el fracaso puede deberse a que, al no tener un conocimiento suficiente sobre la innovación, se acabe delegando en los trabajadores del ayuntamiento que poseen este conocimiento, los cuales adolecen, por el contrario, de falta de autoridad y de los recursos necesarios para crear el mejor clima.

En el caso de la A21L, aunque la decisión de adoptarla provenga del alcalde y del resto del equipo de gobierno, la implementación recae en otros puestos de la organización (ya sea el concejal de Medio Ambiente, el técnico de Medio Ambiente, agentes de desarrollo local, etc.) que tienen un conocimiento mayor, pero que pueden no poseer 
ni la autoridad ni los recursos suficientes para potenciar el buen clima citado. Por ello, el apoyo del equipo de gobierno debe ser activo en todo momento, resaltando la importancia de la A21L para el ayuntamiento y el municipio, comprometiéndose seriamente con ella. En general, el apoyo de los gerentes a la implementación de la innovación está relacionado significativa y positivamente con la calidad de la implementación de políticas y prácticas de una organización (Klein et al., 2001).

En la investigación realizada, los entrevistados, en general, sostienen que el equipo se ha comprometido seriamente en la implementación exitosa de la A21L. No creen que el equipo de gobierno demuestre poco interés por la A21L y están seguros de que la A21L ayudará al ayuntamiento. A su vez, el apoyo a la implementación mejora el clima del ayuntamiento.

\section{Proposición 6: la participación de los ciudadanos y otros grupos de interés en los procesos de A21L influye positivamente en la implementación del plan}

Uno de los resultados más consistentes en la investigación sobre el cambio es que la participación en el mismo tiende a reducir la resistencia (Nadler, 1988; Lines, 2004). La participación puede conducir a una resistencia menor en tanto que: primero, los procesos participativos proveen a la dirección de un marco para explicar y presentar los argumentos del cambio propuesto. Durante este proceso, los motivos para cambiar y la necesidad de cambio se hacen explícitos y tanto los empleados del ayuntamiento como los ciudadanos pueden presentar sus argumentos con objeto de que sean discutidos e incorporados al contenido y proceso de cambio. Segundo, la participación puede dar a los empleados y los ciudadanos influencia directa sobre el contenido del cambio, tanto en la programación de subprocesos como en la gestión de aquellos aspectos del mismo que pueden suponer cambios en la situación actual de ambos grupos (Lines, 2004).

La participación es una variable que, de hecho, se considera clave en la eficacia de la A21L. El documento de Agenda 21 aprobado en 1992 ponía particular énfasis en la necesidad de implicar a todos los sectores sociales y a los ciudadanos en la promoción del desarrollo sostenible. También la participación ciudadana es uno de los principios de la toma de decisiones recogidos por el ICLEI (el Consejo Internacional para las Iniciativas Ambientales Locales, en inglés The International Council for Local Environmental Initiatives) junto con la democracia, la subsidiariedad, la responsabilidad y la transparencia (Hewitt, 1998). Se aconseja que desde el inicio se cree un foro o consejo de medio ambiente que implique a todos los sectores de la comunidad.

La aplicación de un proceso integrador y participativo conlleva una nueva manera de tomar decisiones y, por ello, requiere un cierto cambio de mentalidad sobre el papel de las instituciones y los ciudadanos (Martell y Querol, 2000). Los ciudadanos están acostumbrados a no conformar las decisiones municipales, salvo eligiendo al partido que gobernará. Los ciudadanos deben estar motivados para participar en la toma de decisiones y comprobar posteriormente que sus decisiones, sus opiniones, sus sugerencias... son tenidas en cuenta y llevadas a la práctica en los casos que así resulte conveniente. 
Young (1996) halló cuatro grandes tipos de estrategias de participación utilizadas por los gobiernos locales británicos, expuestas por Martell y Querol (2000) de la siguiente forma: la estrategia ascendente (bottom-up) tiene por objetivo que la comunidad posea el proceso, es decir, el gobierno local está interesado en capacitar a la gente, dándoles un papel real en dar forma a las decisiones y adopta una postura de escucha y aprendizaje. La estrategia "Sí... pero..." es empleada por los gobiernos locales que hacen accesible la participación para que los participantes tengan un papel real en dar forma a las decisiones, pero mantienen el control. En la estrategia de diálogo limitado, los gobiernos se interesan por conocer la reacción sobre algunos detalles de las propuestas. En la estrategia descendente (top-down) sólo se proporciona información al público y a las partes interesadas. Sería deseable una apuesta por una estrategia ascendente donde se implicara a la comunidad y a los diferentes departamentos o concejalías del ayuntamiento.

Según nuestra investigación, los actores sociales que han participado son, por orden, los siguientes: trabajadores del ayuntamiento, grupo político en el poder, ciudadanos, asociaciones de vecinos, grupos ecologistas y asociaciones profesionales y empresariales, sector educativo, resto de partidos políticos, arquitecto municipal, ONG's y sindicatos y, en último lugar, con sólo 8 alusiones, la universidad. En todo caso, se declara bastante unánimemente que unos u otros agentes de la comunidad local han venido participando en la A21L desde el principio (Figura 5).

FIGURA 5 | Agentes que han participado en la A21L

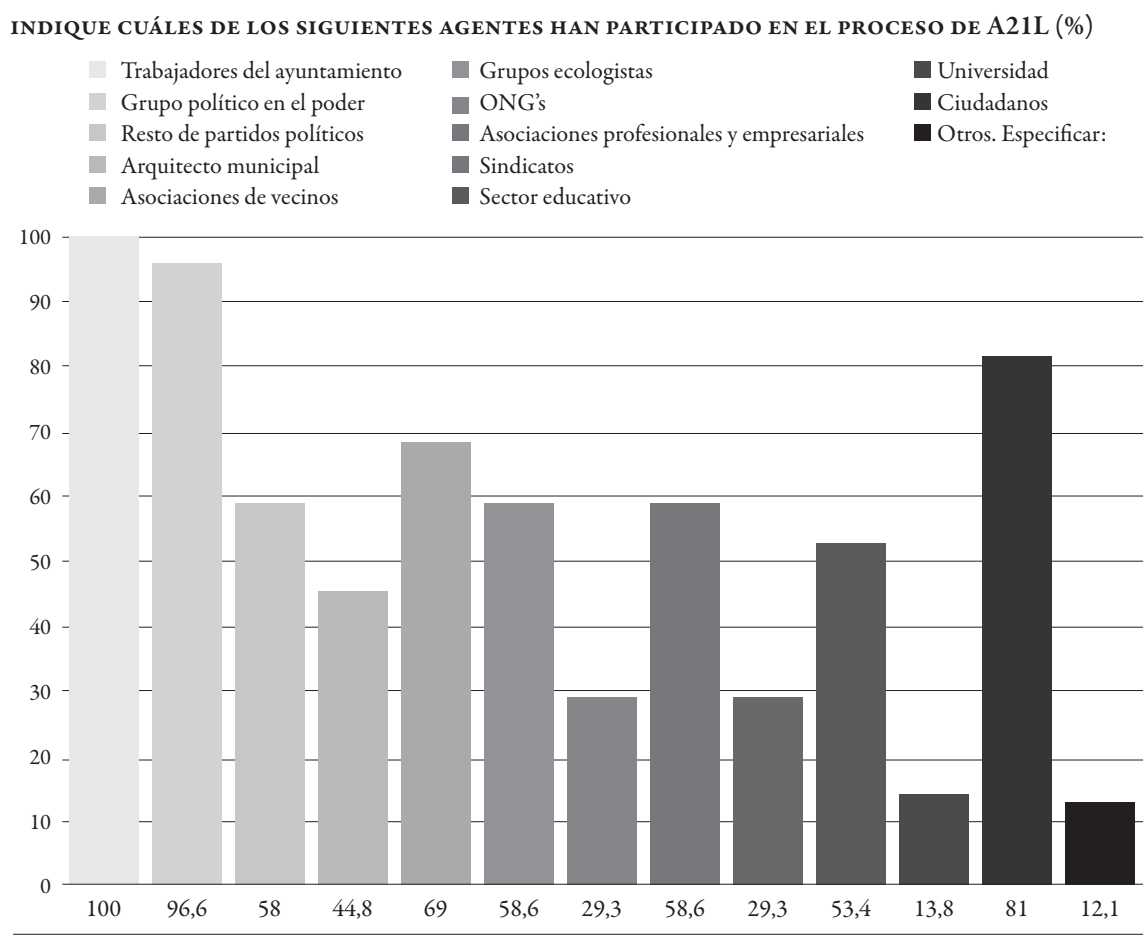

FUENTE ELABORACIÓN PROPIA. 
Además, según los resultados, en aquellos ayuntamientos donde las sugerencias de los actores sociales son tenidas en cuenta con seriedad, la participación ciudadana no ha planteado problemas, los participantes comprenden la complejidad de los temas tratados en los foros y se alcanzan mayores porcentajes de resultados de la implementación (Figura 6). Como nos confirmaba también uno de los entrevistados, "es necesario evitar procesos impuestos". La implementación de la agenda tiene una repercusión evidente en la vida cotidiana del municipio y afecta a cómo toman decisiones todos los grupos de interés del mismo. Lograr impregnar al municipio con los valores que promulga la $\mathrm{A} 21 \mathrm{~L}$ requiere hacer partícipes del proceso de implementación a esos grupos de interés. De hecho, cuando se les pidió a los entrevistados que definieran por orden de prioridad los obstáculos que la implementación de la agenda encontraba en el municipio, los obstáculos sociales fueron los primeros, seguidos por los económicos y políticos.

FIGURA 6 | Estrategia de participación ciudadana utilizada por el ayuntamiento en la $\mathrm{A} 21 \mathrm{~L}$

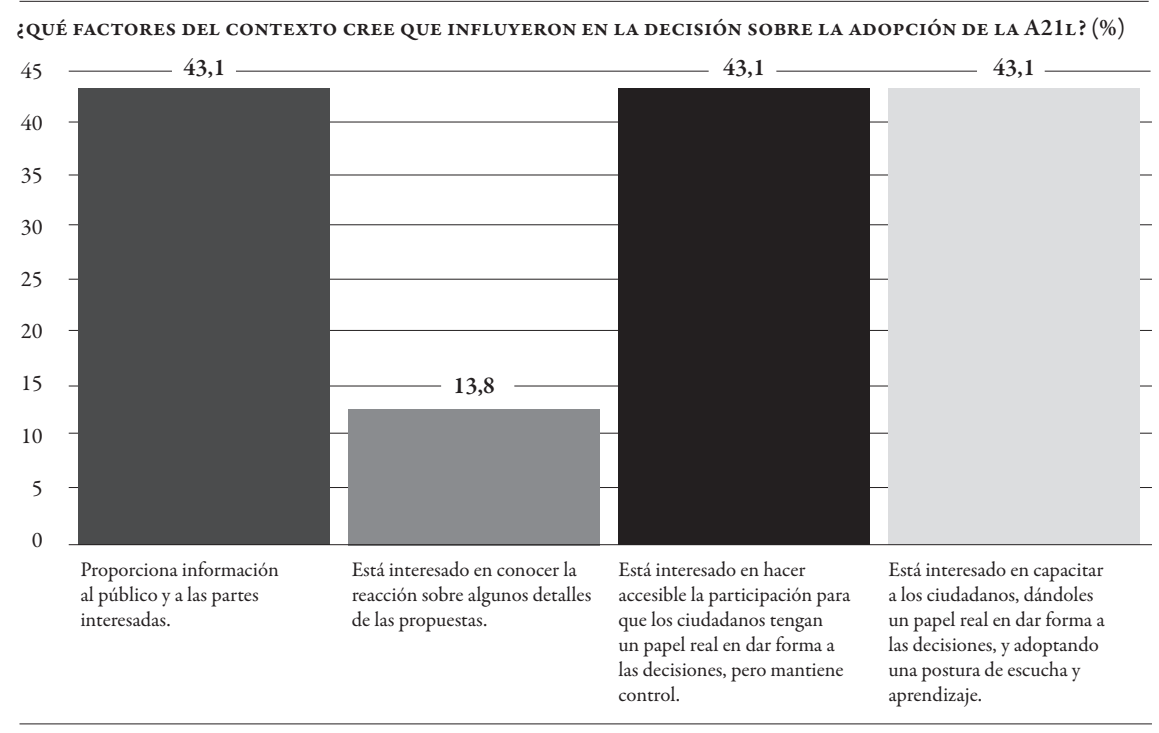

FUENTE ELABORACIÓN PROPIA.

De acuerdo con la investigación realizada, las proposiciones previas describen los factores contextuales y organizacionales que favorecen la adopción e implementación de la A21L por los ayuntamientos. Además, si contemplamos el proceso de implementación de la agenda más de cerca, se pueden describir algunas proposiciones adicionales para explicar lo que hace que la innovación progrese con éxito en el día a día del ayuntamiento. 


\section{Proposición 7: la implementación de la A21L necesita comunicación y coordina- ción en el seno de la organización, y entre ésta y su entorno}

La A21L tiene como finalidad lograr un equilibrio en los criterios de decisión sociales, medioambientales y económicos dentro del ayuntamiento, con objeto de poner en marcha en su comunidad actuaciones eficaces en los tres ámbitos. Es difícil entender que el ayuntamiento como un todo pueda satisfacer este triple criterio de decisión si la visión de la A21L no impregna las decisiones y actuaciones de cada uno de sus departamentos/concejalías. Como los investigadores pudieron observar, la ausencia de una adecuada comunicación y coordinación entre departamentos fue una de las variables más mencionadas por los entrevistados como causa de un menor avance en el proceso de implementación de la innovación. Por otra parte, la comunicación y el intercambio entre municipios en diferentes niveles de avance constituirían un fondo de experiencias que, con seguridad, evitará obstáculos, sorpresas o contratiempos. Cuando se trataba de adoptar la A21L, ha cundido el ejemplo de otras experiencias municipales, por lo que recomendamos divulgar el avance y favorecer el intercambio de experiencias entre municipios. Además, podría ser beneficioso que algún/os miembro/s del ayuntamiento asumiera un papel de enlace o interlocutor entre éste y su entorno. Es necesario para la implementación con éxito de la agenda conseguir aceptación dentro de la organización y, también, del entorno, de ciudadanos a título particular y de grupos de interés, y también apoyo, como recursos económicos, información, apoyo técnico, experiencias ajenas, etc.

\section{Proposición 8: la innovación necesita de una estructura que aglutine tareas, perso-} nas y responsables con estabilidad y profesionalidad

Un análisis más detallado sobre cuáles podían ser los motivos para esa falta de comunicación e integración entre departamentos del ayuntamiento a la que se hacía referencia en la proposición anterior, puso de manifiesto otras carencias que estaban obstaculizando el proceso de implementación de la A21L. Por una parte, en 12 de los ayuntamientos analizados la A21L carecía de una estructura propia y ubicación clara en el organigrama del ayuntamiento. Sólo en uno de los ayuntamientos estudiados se había creado una estructura formal con la única finalidad de responder de la eficacia en la implementación de la A21L, con un equipo fijo y dedicado a temas de A21L. En otro de los ayuntamientos se disponía de una propuesta de estructura organizativa para la A21L. Podemos destacar una interesante diferencia entre ambos diseños estructurales, mientras que en el primer caso la "Oficina Técnica de A21L” forma parte de la jerarquía del ayuntamiento a través de su integración formal en la Concejalía de Medio Ambiente, en el segundo caso se propone crear una organización específica para la A21L que opere de manera paralela a la estructura formal del ayuntamiento, integrando tanto a miembros del mismo como a los grupos de interés externos relacionados con la A21L.

Una estructura clara y definida evitaría que hubiese municipios en los que aún no se conoce la A21L en todas las concejalías. Habría que integrar la A21L en la gestión municipal. Ciertamente este es un tema pendiente a tenor del alto porcen- 
taje de entrevistados que considera que la A21L opera de forma paralela a la gestión municipal y no de forma integrada. De los ayuntamientos encuestados, un $47 \%$ cree que la A21L opera en paralelo frente a un 54\% que percibe a la A21L integrada en la gestión municipal. Como se observa en la figura siguiente (Figura 7), son numerosos los beneficios de una gestión integrada. Consideramos que la estructura organizativa serviría como dinamizador del proceso tanto dentro del ayuntamiento como fuera del mismo.

FIGURA 7 | Gestión integrada frente a gestión paralela en los ayuntamientos

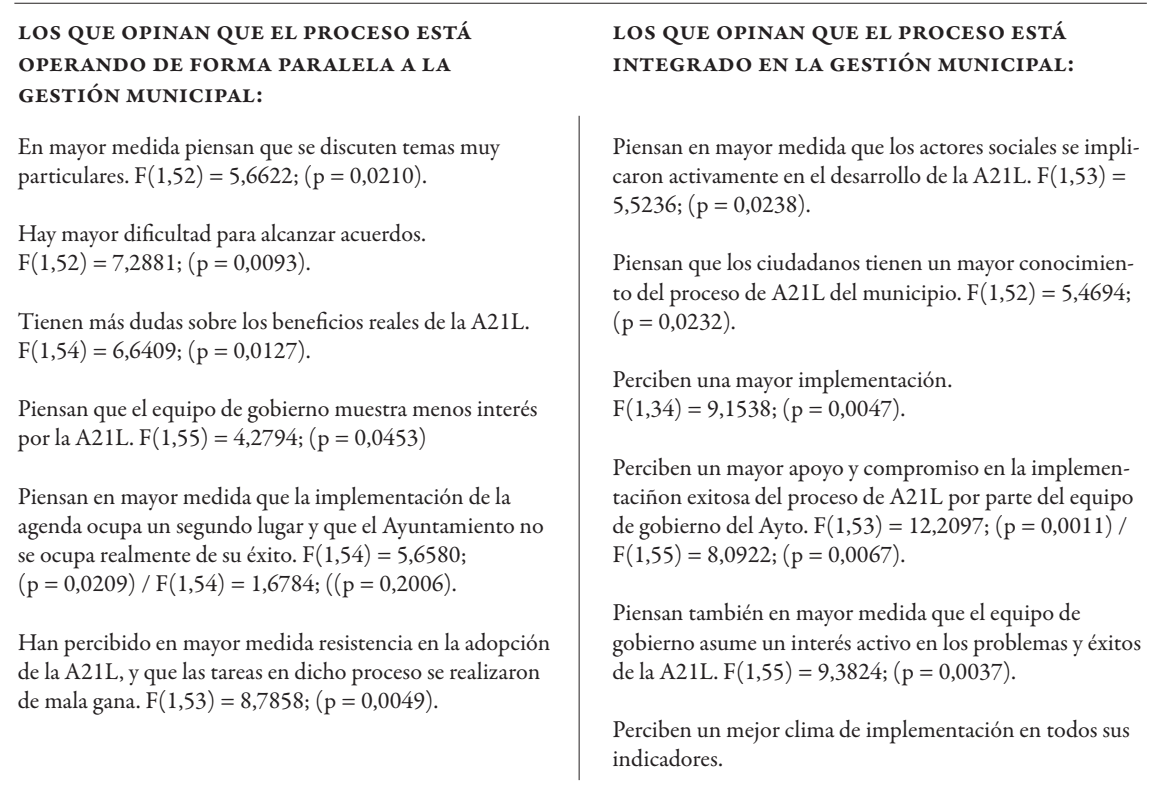

FUENTE ELABORACIÓN PROPIA.

\section{Proposición 9: la A21L ha de ser incluida en la planificación estratégica y a largo plazo del ayuntamiento}

Por otra parte, y en esto coincidían todos los entrevistados, para una mayor coordinación entre departamentos en la implementación de la A21L, ésta ha de ser incluida en la planificación estratégica y a largo plazo del ayuntamiento. Además, es necesaria una mayor implicación del máximo responsable del ayuntamiento en el proceso, no solamente impulsando el mismo, sino también legitimándolo y moderándolo cuando sea necesario y mediante la creación de incentivos para el cambio. No cabe duda de que la motivación para la innovación necesita atención. La integración de la A21L en la estructura organizativa y en el plan estratégico del ayuntamiento ayudaría a integrar el significado de la agenda en el mismo pues, según nos la definió uno de los entrevistados: "la agenda es trabajo técnico más apoyo político”. Por otro lado, esta integración de la agenda en los planes a largo 
plazo del ayuntamiento demanda el diseño de un sistema de control que permita medir la eficacia o grado de cumplimiento de los objetivos de sostenibilidad que contempla la A21L.

\section{Proposición 10: es necesario crear una cultura favorecedora de la implementación} con éxito de la A21L

La naturaleza de la A21L es de gestión compleja. Es un instrumento de principios incuestionables pero que carece de poder. La eficacia de la misma depende de su capacidad de influencia, y ésta será mayor si el contexto en el que ha de operar es receptivo a los principios que defiende. En la medida en que estos principios vayan siendo asumidos y compartidos por más personas tanto dentro del ayuntamiento como en la comunidad local, se irá creando una cultura favorecedora de la implementación con éxito de la A21L. Una cultura que ha de quedar impregnada en la corporación local con independencia del equipo que gobierne durante determinado periodo de tiempo.

Proposición 11: la implementación de la innovación está siendo eficaz en los ayuntamientos estudiados

Según nuestros resultados, existe una asociación positiva entre el porcentaje de implementación del plan de acción y los resultados pretendidos con la misma, poniendo de manifiesto que el diseño y la implementación de planes están siendo eficaces en el logro del desarrollo sostenible como objetivo común en todos los ayuntamientos (Figura 8).

FIGURA 8 | Eficacia en la implementación de la innovación

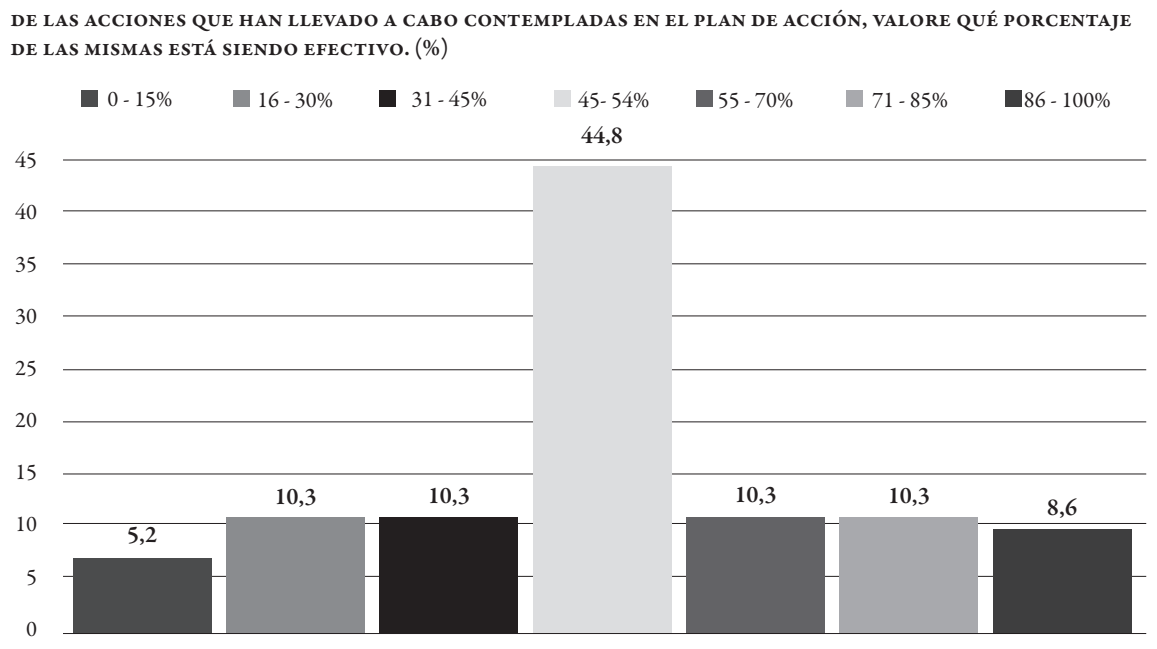

FUENTE ELABORACIÓN PROPIA. 


\section{Conclusiones y recomendaciones para la gestión}

Desde 1992, cuando en la Cumbre de Río nació la Agenda 21, son cada vez más los ayuntamientos que han decidido implantar la A21L con el objetivo de hacer más sostenibles los pueblos y ciudades del mundo. En este trabajo nos hemos centrado en una región concreta, Andalucía, en la que hemos analizado cómo se están llevando a cabo estos procesos. Para ello, hemos definido la A21L como una innovación introducida dentro de una realidad social particular, el ayuntamiento, y que requiere para su implementación una serie de actuaciones que tendrán como finalidad beneficiar al ayuntamiento, a los ciudadanos y al municipio en general.

El trabajo de investigación realizado ha permitido describir y profundizar en el estado actual en que se encuentran las A21L en los municipios andaluces, y presentar como resultado un modelo de proceso de innovación para estos ayuntamientos.

De acuerdo con nuestra investigación la adopción e implementación eficaz de la A21L por los ayuntamientos requiere la identificación y gestión de una serie de variables internas, externas y de proceso, con objeto de lograr que la idea de la sostenibilidad se impregne en el ayuntamiento y en el municipio, y se logren objetivos sostenibles.

- En cuanto a las variables internas, el apoyo a la innovación por parte del equipo de gobierno en el ayuntamiento, la receptividad al cambio por parte de los miembros del ayuntamiento y el clima favorable a la implementación de la agenda se han observado fundamentales para la eficacia de la innovación. Además, es necesario destacar la participación ciudadana y de los diferentes grupos de interés como el eje y centro de la A21L y, por tanto, clave en la realización de sus propósitos más fundamentales.

- En cuanto a las variables externas, concretamente el entorno institucional en el que los ayuntamientos operan, influye en el proceso de innovación. De acuerdo con nuestra investigación, la teoría institucional es un adecuado marco teórico a tener en cuenta al estudiar los procesos de cambio e innovación en los ayuntamientos. En nuestro caso, las presiones coercitivas, normativas y miméticas que recoge esta teoría describen y definen el proceso de adopción de la A21L como innovación.

- Por último, la gestión con éxito de la implementación del proceso de innovación requiere comunicación y coordinación en el seno del ayuntamiento, de una estructura organizativa que defina tareas y responsabilidades, y de la integración de la agenda en los procesos de planificación y control a largo plazo del ayuntamiento. Destacamos, además, la necesidad de una cultura para la innovación por parte de los responsables de la organización. Una cultura que ha de quedar impregnada en la corporación local con independencia del equipo que gobierne durante determinado período de tiempo. 
Esperamos que el modelo propuesto ayude a los ayuntamientos a trabajar por y para la sostenibilidad, por las repercusiones que las decisiones de estas organizaciones pueden tener para las generaciones futuras y que, a la vez, consigamos un avance en los estudios regionales sobre innovación. IEURE

\section{Referencias bibliográficas}

Angle, H., \& Van de Ven, A. (1989). Suggestions for managing the innovation journey. En A. H. Van de Van, R.

Beckhard \& R. T. Harris. Organizational Transitions. Estados Unidos: Addison-Wesley.

Beer, M. (1988). The critical path for change: Keys to success and failure in six companies. En G.H. Brundlant. Nuestro Futuro Común. Madrid, Alianza.

Burnside, R. M. (1990). Improving corporate climates for creativity. En M. A. West \& J. L. Farr (Eds.), Innovation and creativity at work, pp. 265-284. New York: Wiley.

Caravaca, I.; González, G. \& Silva, R. (2005). Innovación, redes, recursos patrimoniales y desarrollo territorial. EURE, Revista Latinoamericana de Estudios Urbano Regionales, 31, 94: 5-24.

Chetty, S. (1996). The case study method for research in small and medium-sized firms. International Small Business Journal, 15: 73-85.

Damanpour, F. (1992). Organizational size and innovation. Organizational Studies, 13, 3: 375-402.

Dimaggio, P.J. \& Powell, W.W. (1983). The Iron Cage Revisited: Institutional Isomorphism and Collective Rationality. American Sociological Review, 48: 147-160.

Dobbin, F.; Sutton, J.; Meyer, J. \& Scott, R. (1993). Equal opportunity law and the construction of internal labor markets. American Journal of Sociology, 99, 2: 396-427.

Dong, L. (2001). Modeling top management influence on ES implementation. Business Process Management Journal, 7, 3: 243-250.

Eisenhardt, K. M. (1989). Building theories from case study research. Academy of Management Review, 14, 4: 532-550.

Eisenhardt, K. M. (1991). Better stories and better constructs: the case for rigor and comparative logic. Academy of Management Review, 16, 3: 620-627.

Eisenhardt, K. M. y Bourgeois III, L, J. (1988). Politics of strategic decision making in high-velocity environments: Toward a midrange theory. Academy of Management Journal 31(4), 737-770.

Font, N. (2000). Local y sostenible. En N. Font \& J. Subirats (Eds.). Local y sostenible. La Agenda 21 Local en España. España: Icaria editorial.

Granovetter, M. (1985). Economic action and social structure: The problem of embeddedness. American Journal of Sociology, 91, 3: 481-510.

Greenwood, R.; Suddaby, R. \& Hinings, C.R. (2002). Theorizing Change: The Role of Professional Associations in the Transformation of Institutionalized Fields. Academy of Management Journal, 45, 1: 58-80.

Gummesson, E. (1988). Qualitative methods in management research. Bromley: Chartwell-Bratt, Studenlitteratur.

Hall, R.H. (1996). Organizaciones, estructuras, procesos y resultados. México: Prentice Hall.

Hewitt, N. (1998). Guía Europea para la Planificación de las Agendas 21 Locales. Editada por el ICLEI para la Campaña Europea de Ciudades y Poblaciones Sostenibles. 
Hitt, M.A., Hoskisson, R.E \& Ireland, R.D. (1990). Mergers and acquisitions and managerial commitment to innovation in M-Form firms". Strategic Management Journal, 11: 29-47.

Holahan, P.J., Aronson, Z. H., Jurkat, M. P. \& Schoorman, F. D. (2004). Implementing computer technology: a multiorganizational test of Klein and Sorra's model. J. Eng. Technol. Management, 21: 31-50.

Kimberly, J. R. \& Evanisko, M. (1981). Organizational innovation: the influence of individual, organizational, and contextual factors on hospital adoption of technological and administrative innovations. Academy of Management Journal, 24: 698-713.

King, N. (1992). Modelling the innovation process: an empirical comparison of approaches. Journal of Occupational and Organizational Psychology, 65: 89-100.

Klein, K.J. \& Knight, A. (2005). Innovation implementation. Current Directions in Psychological Science, 14, 5: 243-246.

Klein, K.J. \& Sorra, J.S. (1996). The challenge of innovation implementation. Academy of Management Review, 21, 4: 1055-1080.

Klein, K.J.; Conn, A.B. \& Sorra, J.S. (2001). Implementing computerized technology: an organizational analysis. Journal of Applied Psychology, 86, 5: 811-824.

Kostova, T. \& Roth, K. (2002). Adoption of an organizational practice by subsidiaries of multinational corporations: Institutional and relational effects. Academy of Management Journal, 45, 1: 215-233.

Leal Millán, A. (1990). Conocer la cultura de las organizaciones: una base para la estrategia y el cambio. Madrid: Actualidad.

Lines, R. (2004). Influence of participation in strategic change: resistance, organizational commitment and change goal achievement. Journal of Change Management, 4, 3: 193-215.

Martell, M. \& Querol, C. (2000). Participación gobierno local y Agenda 21 Local. En N. Font y J. Subirats (Eds.). Local y sostenible. La Agenda 21 Local en España, pp. 65-91. Barcelona: Icaria.

Méndez, R. (2002). Innovación y desarrollo territorial: algunos debates teóricos recientes. EURE, Revista Latinoamericana de Estudios Urbano Regionales, 28, 84: 63-84.

Meyer, J.W. y Rowan, B. (1977). Institutionalized Organizations: Formal Structure as Myth and Ceremony. American Journal of Sociology, 83: 340-363.

Muñoz, Á. (2000). El tamaño organizacional como determinante de innovación. En E. Díez de Castro et al. (Eds.). La pequeña y mediana empresa en el umbral del siglo XXI, pp. 259-269. Granada: Granada University.

Muñoz, A. (2001). La organización innovadora. Sevilla: Mergablu.

Nadler, D. A. (1988). Concepts for the management of organizational Change. En M. L. Tushman y W. L. Moore (Eds). Readings in the managemente of innovations, pp. 718-731. New York: Harper Business.

Nadler, D. A. \& Tushman, M.L. (1989). Leadership for organizational change. En A.M. Mohrman, S.A. Mohrman, G.E. Ledford, T.G. Cummings \& E.E. Lewler (Eds.). Large-scale organizational change. San Francisco: Jossey-Bass.

Nord, W. R. \& Tucker, S. (1987). Implementing Rutine and Radical Innovations. Lexington, MA: Lexington Books.

Pardo del Val, M. \& Martínez Fuentes, C. (2005). Resistencias al cambio organizativo: Un análisis empírico en cambios reactivos y anticipativos. Management, 8, 3: 47-67. 
Rodríguez del Bosque, I. (1999). La construcción del cuestionario. En F.J. Sarabia (Ed.). Metodología para la investigación en marketing y dirección de empresas. Madrid: Editorial Pirámide.

Rogers, E. M. (1983). Diffusion of innovations. New York: Free Press.

Sánchez, F. \& Moura, R. (2005). Ciudades-modelo: estrategias convergentes para su difusión internacional. EURE, Revista Latinoamericana de Estudios Urbano Regionales, 31, 93: 21-34.

Schneider, B.; Gunnarson, S. K. \& Niles-Jolly, K. (1994). Creating the climate and culture of success. Organizational Dynamics, 23, 1: 17-29.

Scott, W.R. (1987). The Adolescence of Institutional Theory. Administrative Science Quarterly, 32: 493-511.

Taylor, J. \& McAdam, R. (2004). Innovation adoption and implementation in organizations: a review and critique. Journal of Genaral Management, 30, 1: 17-38.

Tolbert, P.S. y Zucker, L.G. (1996). The institucionalization of Institutional Theory. En S.R. Clegg, C. Hardy \& W. Nord (Eds.). Handbook of organizational studies. London: Sage.

Van de Ven, A. H. (1986). Central problems in the management of innovation. Management Science, 32: 590-607.

Van de Ven, A. H.; Angle, H., y Poole, M. S. (1989). Research on the management of innovation: The Minnesota studies. New York: Oxford University Press.

Van de Ven, A. H. \& Poole, M. S. (1989). Methods for studying innovation processes. En A. H. Van de Ven, H. Angle \& M. S. Poole. Research on the management of innovation, 1: 31-53. New York: Ballinger/Harper and Row.

Van de Ven, A. H. \& Poole, M. S. (1990). Methods for studying innovation. Development in the Minnesota Innovation Research Program. Organizational Science, 1, 3: 313-335.

Winchester, L. (2006). Desafíos para el desarrollo sostenible de las ciudades en América Latina y El Caribe. EURE, Revista Latinoamericana de Estudios Urbano Regionales, 32, 96: 7-25.

Yin, R. K. (1984). Case study research: Design and methods. Beverly Hills, CA: Sage.

Yin, R. K. (1989). Case study research: Design and methods. California: Sage.

Young, S.C. (1996). Promoting participation and community-based partnerships in the context of Local Agenda 21: A report for practitioners. Manchester: Manchester University.

\section{Referencias electrónicas}

http://www.juntadeandalucia.es/iea/ 